\section{International Scientific Journal Theoretical \& Applied Science}

p-ISSN: 2308-4944 (print) $\quad$ e-ISSN: 2409-0085 (online)

Year: $2017 \quad$ Issue: $04 \quad$ Volume: 48

Published: 14.04.2017 http://T-Science.org

SECTION 8. Architecture and construction.
Grittel Gennadievna Shevchenko

Assistant KubSTU

Dmirtriy Andreevich Gura Candidate of Technical Sciences, Senior Lecturer KubSTU

Ivan Dmitrievich Muranov Bachelor, Student KubSTU

ivanmuranov595@gmail.com

Elizaveta Nikolaevna Bahtarova Bachelor, Student KubSTU

\title{
PROVIDING HIGH-PRECISION MEASUREMENTS BY ELECTRONIC TACHEOMETERS
}

Abstract: Different topographic and geodesic works in all engineering spheres of activities require compliance with different requirements for the accuracy of measurements. High-precision works include determining deposits of a construction, creation of planned high-rise grounding, creation of government geodesic networks and supplementing construction of complex objects (bridges, multi-story buildings and skyscrapers). These works require linear submillimeter and angular sub-second accuracy. It is necessary to know all possible ways of providing precise measurements and then effectively put them to practice. We must also choose appropriate geodesic equipment for conducting high-precision measurements on the area. Moreover, it is essential to identify all external factors which affect the quality of measurements and be able to exclude them.

Key words: geodesy, tacheometer, angular measurements, TOPCON.

Language: Russian

Citation: Shevchenko GG, Gura DA, Muranov ID, Bahtarova EN (2017) PROVIDING HIGH-PRECISION MEASUREMENTS BY ELECTRONIC TACHEOMETERS. ISJ Theoretical \& Applied Science, 04 (48): 64-69.

Soi: http://s-o-i.org/1.1/TAS-04-48-10 Doi: crossef https://dx.doi.org/10.15863/TAS.2017.04.48.10

\section{ОБЕСПЕЧЕНИЕ ВЫСОКОТОЧНЫХ ИЗМЕРЕНИЙ ЭЛЕКТРОННЫМИ ТАХЕОМЕТРАМИ}

Аннотация: Различные топографо-геодезические работы во всех сферах инженерной деятельности требуют соответствия различным требованиям $к$ точности линейно-угловых измерений. Такие высокоточные работы, как определение осадок сооружений, создание планово-высотного обоснования, создание фрагментов государственных геодезических сетей (ГГС), сопровождение строительства ответственных и сложных объектов (мосты, путепроводы, многоэтажные дома и небоскребы), требуют линейную субмиллиметровую и угловую субсекундную точность. Необходимо знать всевозможные способы обеспечения высокоточных измерений и уметь выполнять их. Также, необходимо уметь выбирать геодезическое оборудование для проведения высокоточных измерений на местности. Помимо всего этого, в обязательном порядке следует знать и выявлять все внешние факторы, влияюшие на качество измерений, а также, уметь их исключать.

Ключевые слова: геодезия, тахеометр, угловые измерения, ТОРСОN

\section{Введение}

Причины погрешностей

Существуют разнообразные причины погрешностей в линейно-угловых измерениях. Самые значительные из них кроются в инструментальных и методических ошибках:

- Отсутствие тщательных установок перед началом работы (приведение прибора в рабочее состояние (горизонтирование и центрирование над пунктом или точкой хода), обеспечение незыблемости штатива).

- Недостаточное количество избыточных измерений, некачественное наведение на предметы

- Невыполнение различных контрольных измерений.

- Выбор неудовлетворяющих пунктов в качестве исходных. 
Также, существуют и другие различные причины потери точности в измерениях: ошибки человеческого фактора и влияние окружающей среды, которые тоже необходимо устранять. [9]

\section{Способы и пути повышения точности:}

\section{1. Исключение инструментальных} ошибок

Перед работой необходимо тщательно выбирать и подбирать используемое геодезическое оборудование. Штатив должен быть устойчивым, ножки его не должны прогибаться, концы ножек должны быть заострены, а становой винт не иметь люфта. Трегерная подставка должна гладко ездить при центрировании, а центрир с цилиндрическим и круглым уровнем должны быть хорошо отъюстированными. Оборудование должно быть высокоточным, таким как Topcon OS-101L или Topcon OS-103L.

В.Л. Ассур излагал следующие погрешности угломерных приборов, которые применимы в настоящее время к современным электронным тахеометрам:

1) Параллакс сетки нитей. Параллакс может давать довольно значительную погрешность. Полностью его устранить невозможно, но следует стремиться к его отсутствию. Для этого глаз наблюдателя должен находиться на продолжении визирной оси.

2) Биссектор сетки нитей. Биссектор должен находиться в коллимационной плоскости. Его следует периодически юстировать, но также, как и параллакс сетки нитей, полностью отъюстировать его не удается. Для устранения ошибки, наводиться на предметы местности следует одной и той же точкой биссектора, близкой к центру поля зрения трубы.

3) Наведение. Наведение на цель наводящими винтами может производиться ввинчиванием («от себя») и вывинчиванием («на себя») винта наводящего устройства. Следует наводиться на цель исключительно ввинчиванием винта наводящего устройства. [8]

\section{2. Исключение методических ошибок}

Во-первых, для обеспечения точности, необходимо делать достаточное количество избыточных измерений, которые уменьшат погрешность в каждом отсчете. Например, при измерении расстояния дальномером, следует брать на один, а пять-шесть отсчетов и брать в обработку среднее значение.

Необходимо выполнять всевозможные контрольные измерения. Например, при проложении хода, следует брать отсчеты при двух кругах - КЛ и КП. Из двух кругов находить коллимационную ошибку: $\mathrm{C}=\left(\right.$ КЛ - КП $\left.\pm 180^{\circ}\right) \times$
2, она не должна превышать двойной точности тахеометра. Для Topcon OS-101L эта величина составляет 2", а для Topcon OS-103L - 6".

На каждой точке необходимо замыкаться на начальную цель. Значение замыкания должно стремиться к нулю. [10]

\section{3. Погрешности, вызванные влиянием} внешней среды.

Очень важную роль в качестве и точности измерений играет влияние внешней среды.

1) Во-первых, внешняя среда напрямую влияет на качество изображения. Условия видимости зависят от прозрачности атмосферы. В приземных слоях атмосферы всегда присутствует дымка, состоящая из мельчайших частиц пыли, дыма и водяных паров. Это влияет, также, и на контрастность изображения. При коэффициенте видимости 0,82 условия видимости считаются удовлетворительными. А при коэффициенте 0,92 условия видимости считаются хорошими, а дальность видимости достигает 40-50 км.

2) Во-вторых, приземленные слои атмосферы из-за различной температуры, давления и влажности имеют различную плотность. Световой луч, обычно, претерпевает различные преломления. Это явление называется рефракцией. При наихудших условиях, рефракция может давать погрешность до 10".

Чтобы исключить влияние рефракции, необходимо:

- Поверхности сырых низменностей и озер пересекать направлениями симметрично, а реки и долины - под прямым углом.

- Вблизи визирного луча не должно лежать никаких предметов, так как любой предмет может нагреваться быстрее, чем воздух, значит и воздух около предмета будет нагреваться быстрее и иметь меньшую плотность.

3) Изменение температуры тахеометра. Особенно наибольшую погрешность дает неравномерное нагревание отдельных его частей. Это ведет к нарушению геометрической схемы, положенной в основу конструкции инструмента, отъюстированную при поверках. [8]

4. Наиболее выгодное время
проведения линейно-угловых измерений
Наиболее выгодным для наблюдений
считается время, когда влияние рефракции
минимальное, изображение
видимость и контрастность наблюдаемых целей
удовлетворительная. По по многолетним
наблюдениям выявено, что это время
начинается утром (через полчаса после восхода)
до 8-9 часов утра. А также, вечерние часы:
начиная с 16 часов и заканчивая за полчаса до
захода солнца. Это обусловливается ежедневным




\begin{tabular}{l|lr|ll|ll} 
& ISRA (India) & $=\mathbf{1 . 3 4 4}$ & SIS (USA) & $=\mathbf{0 . 9 1 2}$ & ICV (Poland) & $\mathbf{= 6 . 6 3 0}$ \\
Impact Factor: & ISI (Dubai, UAE) $=\mathbf{0 . 8 2 9}$ & PUHL (Russia) $=\mathbf{0 . 2 3 4}$ & PIF (India) & $=\mathbf{1 . 9 4 0}$ \\
& GIF (Australia) & $=\mathbf{0 . 5 6 4}$ & ESJI (KZ) & $=\mathbf{3 . 8 6 0}$ & IBI (India) & $=\mathbf{4 . 2 6 0}$
\end{tabular}

равномерным нагреванием и остыванием воздуха, которое влияет практически на все факторы стабильности работы прибора. [8]

\section{Выбор используемого оборудования}

Тахеометры подразделяются на 3 типа: инженерные, технические и роботизированные (рис. 1-4). Когда разговор идет о точности, то подразумеваются роботизированные

инженерные тахеометры. Сравнительная характеристика наиболее точных на данный момент тахеометров представлена в таблице 1 . Образцами таких тахеометров являются следующие модели:

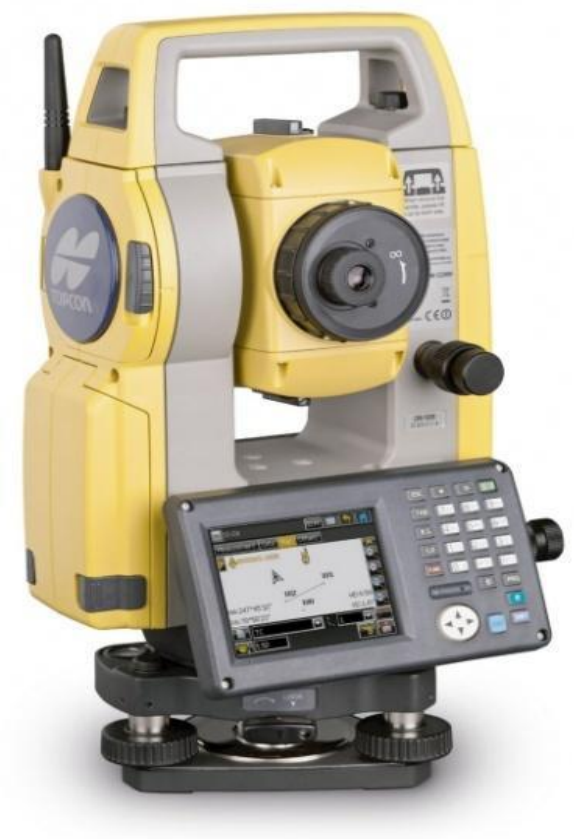

Рисунок 1 - Topcon OS-101L.

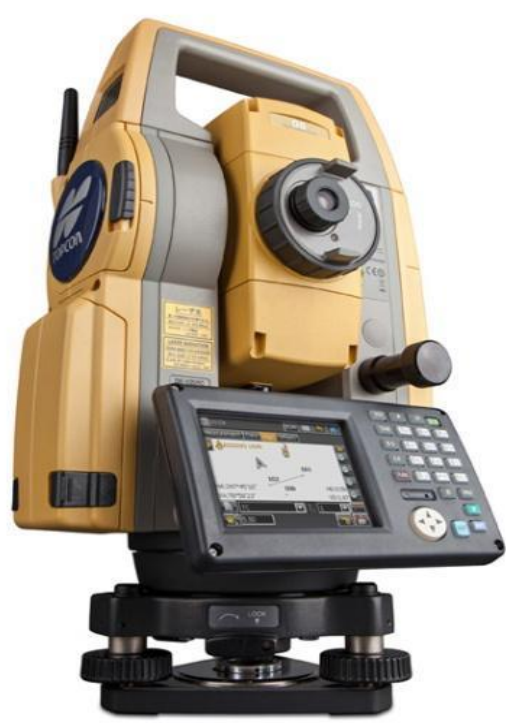

Рисунок 2 - Topcon DS-101 


\begin{tabular}{|c|c|c|c|c|c|c|}
\hline Impact Factor: & $\begin{array}{l}\text { ISRA (India) } \\
\text { ISI (Dubai, UAE } \\
\text { GIF (Australia) } \\
\text { JIF }\end{array}$ & $\begin{array}{l}=1.344 \\
=0.829 \\
=0.564 \\
=1.500\end{array}$ & $\begin{array}{l}\text { SIS (USA) } \\
\text { PИНЦ (Russia) } \\
\text { ESJI (KZ) } \\
\text { SJIF (Morocco) }\end{array}$ & $\begin{array}{l}=0.912 \\
=0.234 \\
=3.860 \\
=\mathbf{2 . 0 3 1}\end{array}$ & $\begin{array}{l}\text { ICV (Poland) } \\
\text { PIF (India) } \\
\text { IBI (India) }\end{array}$ & $\begin{array}{l}=6.630 \\
=1.940 \\
=4.260\end{array}$ \\
\hline
\end{tabular}

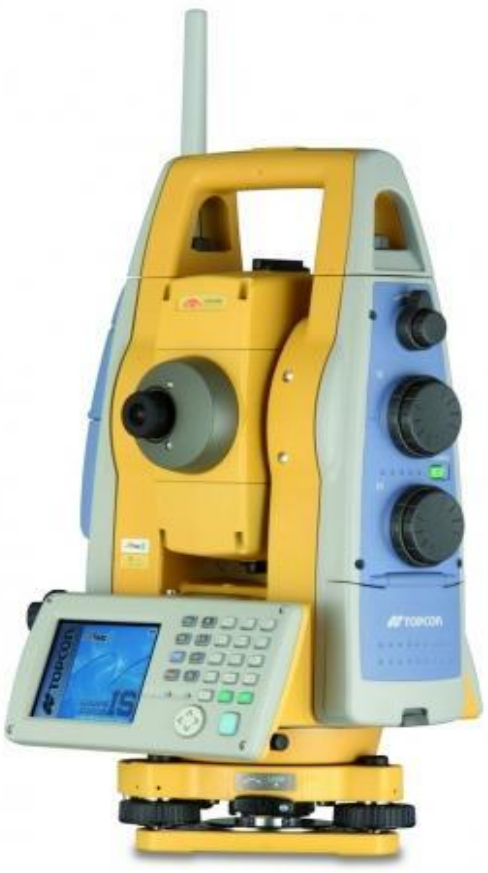

Рисунок 3 - Topcon IS-301.

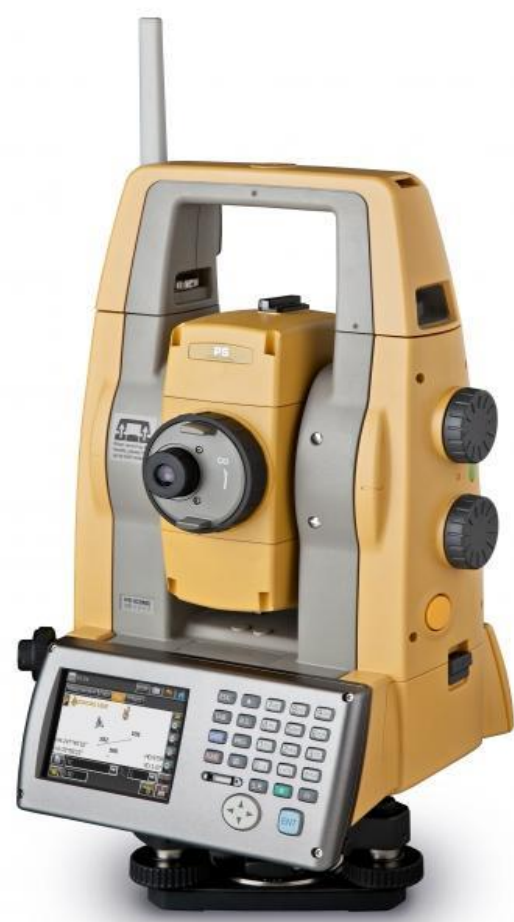

Рисунок 4 - Topcon PS-101. 


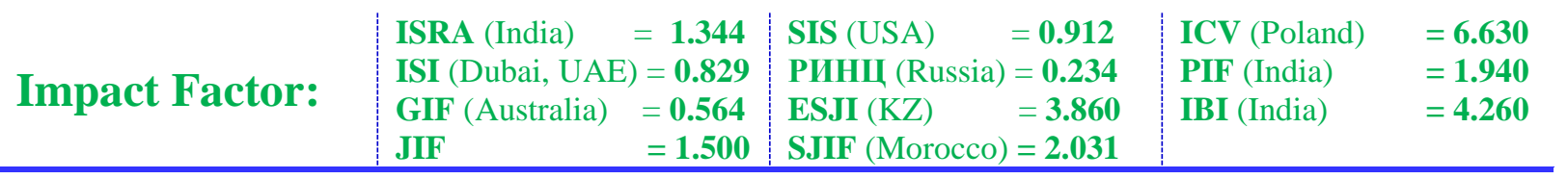

Сравнительная характеристика тахеометров Tорсоп

Таблица 1

\begin{tabular}{|c|c|c|c|c|}
\hline & OS-101L & DS-101 & IS-301 & PS-101 \\
\hline Класс прибора & Инженерный & Роботизированный & Роботизированный & Роботизированный \\
\hline СКО измерения угла & $1 "$ & $1 "$ & $1 "$ & $1 "$ \\
\hline $\begin{array}{c}\text { Дальность взятия } \\
\text { отсчета по } \\
\text { дальномеру в б/о } \\
\end{array}$ & $0.3-500 \mathrm{M}$ & $0-1000$ м & $5-2000 \mathrm{M}$ & $0,3-1000 \mathrm{м}$ \\
\hline $\begin{array}{c}\text { Дальность взятия } \\
\text { отсчета по } \\
\text { дальномеру на пленку }\end{array}$ & $0.3-500 \mathrm{M}$ & $0-1000 \mathrm{~m}$ & $5-2000 \mathrm{M}$ & $0,3-1000$ м \\
\hline $\begin{array}{c}\text { Дальность взятия } \\
\text { отсчета по } \\
\text { дальномеру на призму }\end{array}$ & $0.3-5000 \mathrm{M}$ & $0-6000 \mathrm{~m}$ & $5-3000 \mathrm{M}$ & $0,3-6000$ м \\
\hline $\begin{array}{c}\text { Точность измерения } \\
\text { расстояний в б/o }\end{array}$ & $\begin{array}{c}3+2 \times 10^{-6} \times \\
\text { D MM }\end{array}$ & $2+2 \times 10^{-6} \times \mathrm{D}$ мм & $10 \mathrm{мm}+10 \mathrm{ppm}$ & $2 \mathrm{Mm}+2 \mathrm{ppm}$ \\
\hline $\begin{array}{l}\text { Точность измерения } \\
\text { расстояний на призму }\end{array}$ & $\begin{array}{c}2+2 \times 10^{-6} \times \\
\text { D мM }\end{array}$ & $1,5+2 \times 10^{-6} \times \mathrm{D}$ мM & $2 \mathrm{~mm}+2 \mathrm{ppm}$ & $1,5 \mathrm{~mm}+2 \mathrm{ppm}$ \\
\hline $\begin{array}{c}\text { Увеличение } \\
\text { зрительной трубы }\end{array}$ & $30 \mathrm{x}$ & $30 \mathrm{x}$ & $30 \mathrm{x}$ & $30 \mathrm{x}$ \\
\hline $\begin{array}{c}\text { Пыле- } \\
\text { влагозащищенность }\end{array}$ & IP65 & IP65 & IP54 & IP65 \\
\hline $\begin{array}{c}\text { Рабочая температура, } \\
{ }^{\circ} \mathrm{C}\end{array}$ & $-30 \ldots+50$ & $-20 \ldots+50$ & $-20 \ldots+50$ & $-20 \ldots+50$ \\
\hline $\begin{array}{c}\text { Точность оптического } \\
\text { центрира, мм }\end{array}$ & $<0.5$ & $<0.5$ & $<0.5$ & $<0.5$ \\
\hline Внутренняя память & $500 \mathrm{Mb}$ & $\begin{array}{c}500 \mathrm{Mb}+\text { съемная до } \\
8 \mathrm{~Gb}\end{array}$ & $1 \mathrm{~Gb}$ & $\begin{array}{c}500 \mathrm{Mb}+\text { съемная до } \\
8 \mathrm{~Gb}\end{array}$ \\
\hline $\begin{array}{c}\text { Период работы при } \\
+20^{\circ} \mathrm{C} \\
\end{array}$ & 20 часов & 5 часов & 3.5 часа & 4 часа \\
\hline Вес,, кг & 5,7 & 6,1 & 6,2 & 6,9 \\
\hline
\end{tabular}

Из таблицы можно сделать вывод, что модели имеют высокую точность измерения расстояний, при этом модель инженерного тахеометра OS-101L имеет меньшую, по сравнению с другими моделями, дальность взятия отчета без отражателя и на пленку. [11]

\section{Заключение}

Таким образом, накапливается очень много факторов, влияющих на потерю точности в линейно угловых измерениях. Также, усугубляет ситуацию то, что все современные приборы построены на электронике, и невозможно проследить за каждым его вычислением. Но следуя всем указаниям, мы исключим все возможности потери точности в измерениях. После рассмотренного выше, напрашивается вывод, что при высокоточных измерениях необходимо делать всевозможные избыточные измерения, различные контроли, исключать все влияющие факторы природного характера. Также, необходимо грамотно выбирать используемое оборудование. И таким образом, обработчик получит грамотные измерения для обработки.

\section{References:}

1. Gura TA, Ereshko PS (2016) Trebovanija k tochnosti vypolnenija geodezicheskih izmerenij pri opredelenii osadok zdanij. V sbornike:
EVROPEJSKIE ISSLEDOVANIJa Mezhdunarodnoj
NAUChNYE sbornik statej nauchno-prakticheskoj 


\begin{tabular}{l|lrl|l|ll} 
& ISRA (India) & $=\mathbf{1 . 3 4 4}$ & SIS (USA) & $=\mathbf{0 . 9 1 2}$ & ICV (Poland) & $=\mathbf{6 . 6 3 0}$ \\
Impact Factor: & ISI (Dubai, UAE) $=\mathbf{0 . 8 2 9}$ & PUHL (Russia) $=\mathbf{0 . 2 3 4}$ & PIF (India) & $=\mathbf{1 . 9 4 0}$ \\
& GIF (Australia) & $\mathbf{0 . 5 6 4}$ & ESJI (KZ) & $=3.860$ & IBI (India) & $=\mathbf{4 . 2 6 0}$ \\
& JIF & $=\mathbf{1 . 5 0 0}$ & SJIF (Morocco) & $=\mathbf{2 . 0 3 1}$ & & \\
\hline
\end{tabular}

konferencii. pod obshhej redakciej G.Ju. Guljaeva. 2016. p. 190-194.

2. Gura TA, Bobuh DN (2016) Sravnitel'naja harakteristika jelektronnyh taheometrov Sokkia, Nikon i Topcon // V sbornike: INTERNATIONAL INNOVATION RESEARCH sbornik statej pobeditelej V Mezhdunarodnoj nauchno-prakticheskoj konferencii. Penza, 2016. p. 170-175.

3. Shevchenko AA, Krivcov JA (2016) TREBOVANIJa ISSLEDOVANIJ JELEKTRONNYH TAHEOMETROV V USLOVIJaH OTSUTSTVIJa SPECIAL"NOJ

LABORATORII // V sbornike: EVROPEJSKIE NAUChNYE ISSLEDOVANIJa sbornik statej Mezhdunarodnoj nauchno-prakticheskoj konferencii. pod obshhej redakciej G.Ju. Guljaeva. 2016. p. 200-203.

4. Zheltko CN, Gura DA, Pastuhov MA, Shevchenko GG (2016) Ob issledovanijah uglomernyh pogreshnostej jelektronnyh taheometrov // Monografija. Krasnodar, 2016, $143 \mathrm{p}$.

5. (1976) GOST 22268-76. Geodezija, terminy i opredelenija.

6. (1988) GOST 23543-88. Pribory geodezicheskie. Obshhie tehnicheskie uslovija.

7. (2001) GOST R 51774-2001. Taheometry jelektronnye. Obshhie tehnicheskie uslovija.

8. Assur VL, et al. (1979) «Vysshaja geodezija», Nedra, Moscow.
9. Kuroshev GD, Smirnov LE (2008) «Geodezija i topografija», IC «Akademija», Moscow.

10. Glinskij SP, et al. (1995) «Geodezija», «Kartgeocentr» - «Geoizdat», Moscow.

11. (2016) Katalog geodezicheskih priborov ZAO «GSI», Sankt-Peterburg.

12. (2016) Katalog geodezicheskih priborov ZAO «Geodezicheskie pribory», Sankt-Peterburg.

13. (2017) Ofical'nyj web-sajt Topcon Corporation Available: https://www.topconpositioning.com/ (Accessed: 10.04.2017).

14. (2017) Oficial'nyj kanal v seti internet proekta California Land Surveyors Association, USA

15. Gura DA (2016) Razrabotka metodov issledovanija jelektronnyh taheometrov $\mathrm{v}$ uslovijah proizvodstva dlja ocenki i povyshenija tochnosti izmerenija gorizontal'nyh uglov / avtoreferat dissertacii na soiskanie uchenoj stepeni kandidata tehnicheskih nauk po special'nosti 25.00.32 Geodezija / Moskovskij gosudarstvennyj universitet geodezii i kartografii. Moscow, 2016. - 24p.

16. Pastuhov MA, Denisenko VV, Gura DA, Shevchenko GG (2016) Opredelenie pogreshnosti geodezicheskih priborov za nepravil'nost' formy capf i bokovoe gnutie zritel'noj truby // Nauchnye trudy Kubanskogo gosudarstvennogo tehnologicheskogo universiteta. 2016. № 11. p. 155-171 